\title{
Dynamic thermograms processing for the optimization and the automation of hydrocarbons pollution on sea surface detection
}

\author{
by G. Distefano*, F. Freni**
}

* IMC Service ${ }^{\circledR}$ S.r.I., Via Macello 18 - 95030 Mascalucia CT, gdistefano@imcservice.eu

** Dept. of Industrial Chemistry and Materials Engineering, University of Messina, Viale F. Stagno D'Alcontres 31- 98166 Messina, Italy, fabfreni@unime.it

\begin{abstract}
In this work, a new thermograms quantitative post-processing method for the optimization of hydrocarbons monitoring on sea surface is presented. The proposed method is based on mathematically filtering and integrating discrete sets of thermal images, acquired during hydrocarbons flowing into the sea from dynamic sources (i.e. boats). It permits to avoid some typical error causes and to detect smaller quantities of pollutant with respect to actual qualitative technique capability. Moreover, the monitoring process automation is implemented, in order to develop a live method, able to notify and to alarm if a too high hydrocarbons level is reached in the area of interest.
\end{abstract}

\section{Introduction}

At the present, the monitoring of pollutant presence on the sea surface is typically carried out by means of some standard methods, among which the on-the-ground water sampling and the visual survey are some examples. Both above mentioned methods show some limits, due to the inability of analyzing wide areas and to the difficulty of detecting small pollution sources. Furthermore, they don't permit to implement an automated method [1].

In last years, several studies have been carried out in order to implement wide areas pollution control techniques, thus the use of qualitative infrared thermography (IT) to monitor the hydrocarbons marine injection has been increased noticeably. Some works [2-3] have developed qualitative methods, showing the technique capability of detecting pollutant pouring on the sea, and demonstrating that the ability of identifying contaminant is due to the different emissivity values of water $\left(\varepsilon_{\text {water }}=0.98\right)$ and gasoline $\left(\varepsilon_{\text {gasoline }}=0.90\right)$. However, the IR thermography pollutant on the sea surface detection is strongly affected by a wide number of variables, that might result in misleading results and, in some case, in the impossibility of performing the analysis. Weather conditions are key factors in the technique implementation, as example the presence of rain can lead to inhomogeneity in thermal image, caused by both the rain - water temperature difference and the sea surface ripples, thus hiding the pollutant presence effect. In addition, also wind and moisture are critical issues for the test results. Other examples of noise source are the wave motion (which is the most important test limiting factor in some conditions), the presence of moving subjects, such as boats or birds. Furthermore, the technique capability is affected by geometrical feature of pollutant film, such as size and thickness [4]. The bigger and thicker the hydrocarbon injection, the better its identification.

In this paper, a new thermograms post-processing method which allows to avoid some of the limitations associated with the existing IT technique is introduced. The proposed method concerns on the acquisition of thermograms at different times after the transit of a pollutant source and on automated algorithms that alter these images in order to avoid noise and to provide a better thermal contrast between polluted areas and surrounding zones. The basic theoretical idea is that as an hydrocarbon film is present on the water surface, it tends to stay compact over time, avoiding dispersions. This stability condition can be exploited by image integration to improve the pollutant identification, regardless of surrounding conditions. Both laboratory and on-field experimental measurements have been carried out in order to analyze different condition, thus defining dynamic integration intervals, updated depending on weather, which allow to minimize the effect of external noise sources.

The main aim of this work is the exploiting of dynamic image integration to implement an automatic system for the hydrocarbons on the sea detection and tracking, avoiding several noise source, which are limiting factors of the technique.

\section{Experimental method}

\subsection{Measurement setup}

The experimental method proposed in our study can be carried out by means of low-cost instrumentation, consisting in a thermal camera and a processing hardware. Hence, the use of a non cooled camera permit to keep down the set up cost. For this reason, the acquisition step has been carried out with a FLIR ThermaCAM ${ }^{\mathrm{TM}}$ SC640 infrared 
camera equipped with a 640 × 480 pixels FPA uncooled microbolometer sensor, working in the 7.5 to $13 \mu \mathrm{m}$ spectral band with a thermal sensitivity of $30 \mathrm{mK}$ at $30^{\circ} \mathrm{C}$.

The performed experimental activity had the main goal of defining the most important influence parameters involving in the automation of hydrocarbons pollution detection. For this reason, the first part of the work has been carried out by laboratory experimental tests, in which ideal conditions were achieved. A water filled test tank of dimensions $0.6 \times 0.7 \times 0.2 \mathrm{~m}(\mathrm{~W} \times \mathrm{L} \times \mathrm{H})$ was used, positioned at a distance of $2 \mathrm{~m}$ from the camera, which was held by a $1 \mathrm{~m}$ tall tripod, with horizontal axis inclined of $22^{\circ}$, in order to simulate real measurement conditions. No reflections sources were involved in the setup, and tests were performed during a sunny day.

A typical experimental test consisted in the acquisition of a sequence of 180 thermograms, at a frequency of 0.5 $\mathrm{Hz}$, thus for a duration of 6 minutes. First 15 steps were acquired in stable condition, and, after these, $2 \mathrm{cc}$ of pollutant (gasoline) were introduced. The complete sequence permitted to understand how the detection process occurs, avoiding every typical noise source involved in this kind of measurements (i.e. waves, boats).

The following activity has been the analysis of sea tests data, with the application of images filtering and integration, in order to study the influence of different parameters, thus optimizing the image post-processing. The last step has been the experimental on-field test method verification.

\subsection{Post processing algorithm}

The proposed algorithm, built up in the Matlab ${ }^{8}$ environment, permits to automatically identify the presence of a pollutant in the water. A flow chart of the algorithm used is reported in fig. 1

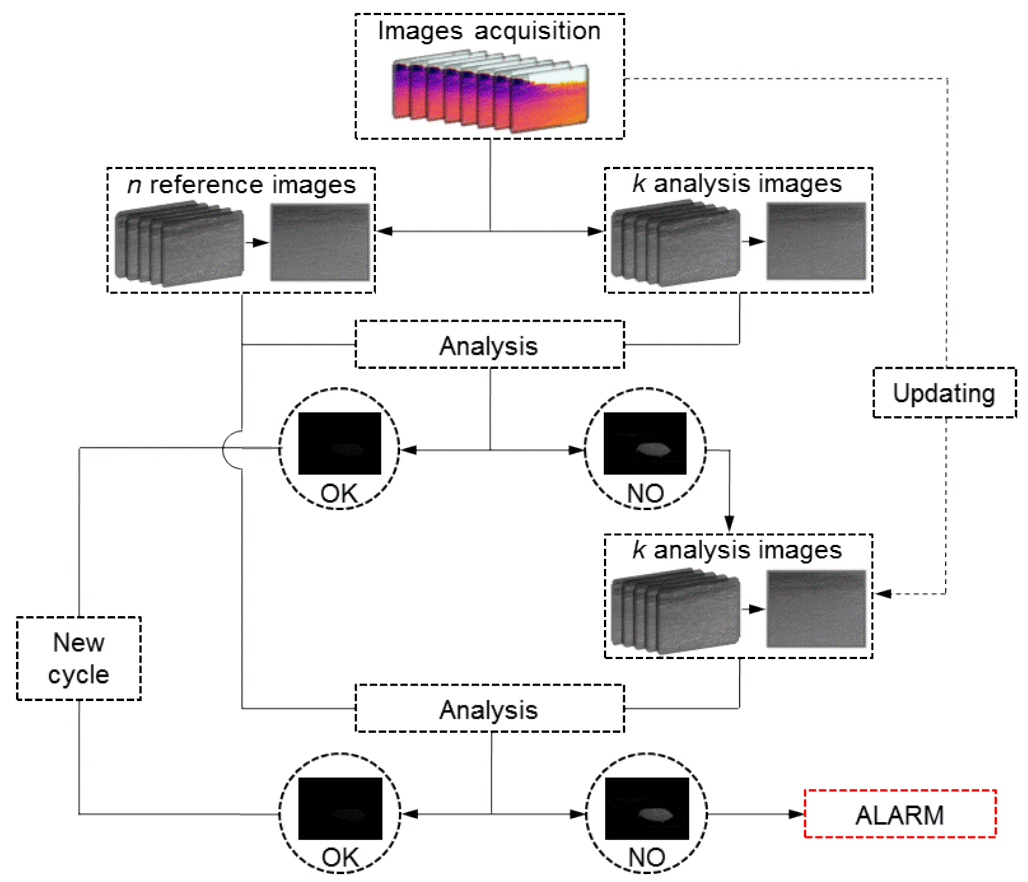

Fig.1. Flow chart of the algorithm used for pollutant identification

Starting from images acquired in the experimental stage (acquisition frequency $0.5 \mathrm{~Hz}$ ), thermograms are live processed by the correlation algorithm. A fundamental phase of the analysis is the definition of the reference image. In the proposed case, the reference image is constantly updated, so as to evolve with the noise environmental sources (clouds, boats, waves). The algorithm acquires images from the thermal camera, applying a predefined region of interest (ROI), it converts images in 8 bit gray level arrays and defines the reference one as the average of last $n$ acquisitions. Using the average of $n$ images as a reference allows to filter typical environmental noise, thanks to its random nature. Therefore the number $n$ directly depends on weather conditions, and increases as the external noise grows up.

At this point, the successive $k$ thermal images are compared with the reference, in order to evaluate the possible presence of hydrocarbons. This is achieved through the reference subtraction from the analysed images, the subsequent application of filters that permit to minimize noise, and the sum of $k$ gradient resulting images. In this case, the sum of $k$ images is performed, in order of both filtering the environmental noise, that statistically tends to comply increasing the $k$ number, and gaining the hydrocarbon presence effect. The successive analysis of the resulting image allows to define the sea surface conditions. If the image analysis does not identify the presence of particular gradients (absence of pollutant), the cycle updates the reference, excluding the first of the $n$ images and inserting the first of the $k$ images just analyzed. Thus, the new acquired thermogram is included in $k$ analysis image, and so on. 
In the event that, on the contrary, the analysis identifies the presence of an hydrocarbons characteristic gradient, the reference is maintained as the previous one for a further cycle, and only the analyzed image is updated. If the image analysis does not show similar gradients as the previous image, the cycle restarts again automatically. If the new image investigation shows similar characteristics to the previous image, an alarm signal is generated, and the event is recorded.

\section{Preliminary results}

Fig. 2 shows the analysis result in the case of ideal conditions, in laboratory without external noise.
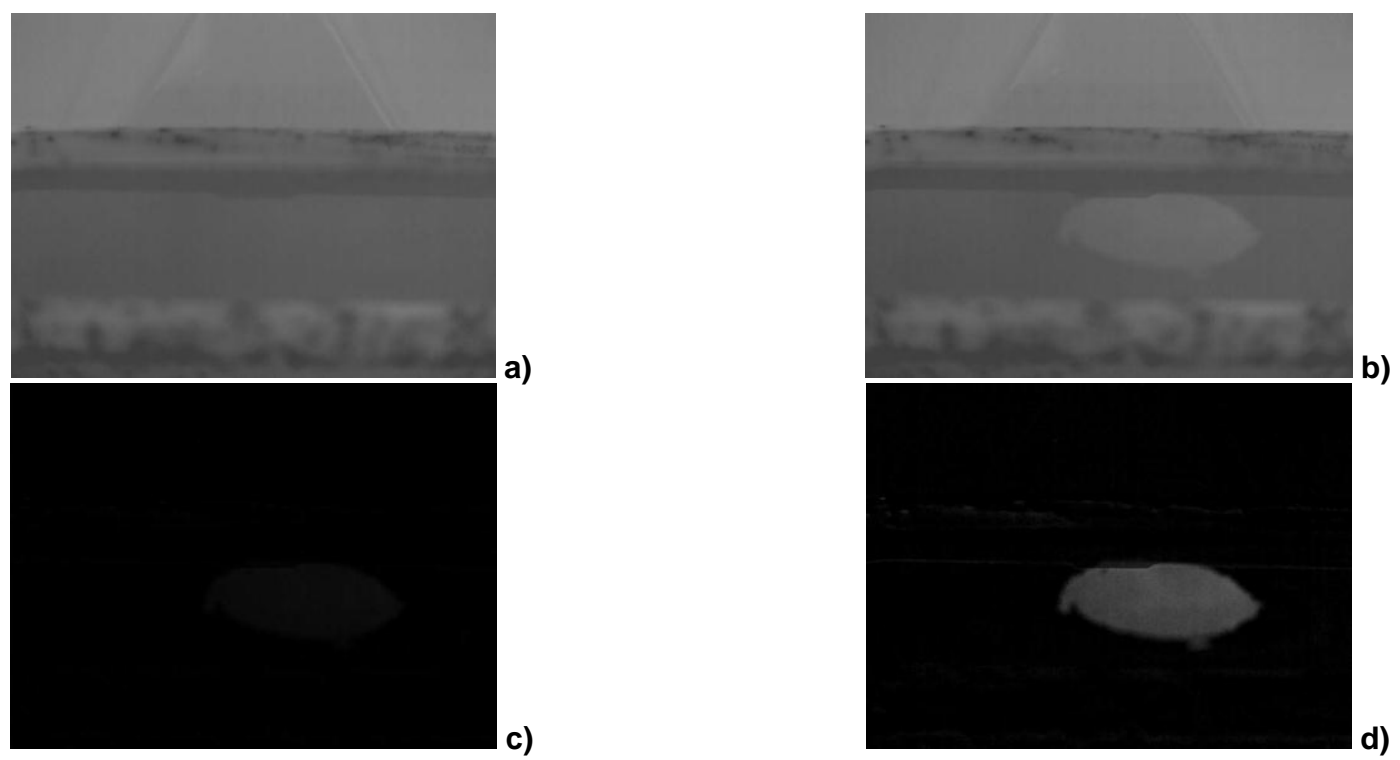

Fig. 2. Infrared and resulting images during the analysis in ideal laboratory conditions. a)averaged reference thermogram; b)thermogram acquisition after hydrocarbon injection; c)result of the single image analysis; d)result of integrated $k$ images analysis.

The integration number has been defined after several laboratory tests, in which a noise source was investigated for each step. In Fig. 3 laboratory results in the case of wind presence are reported.
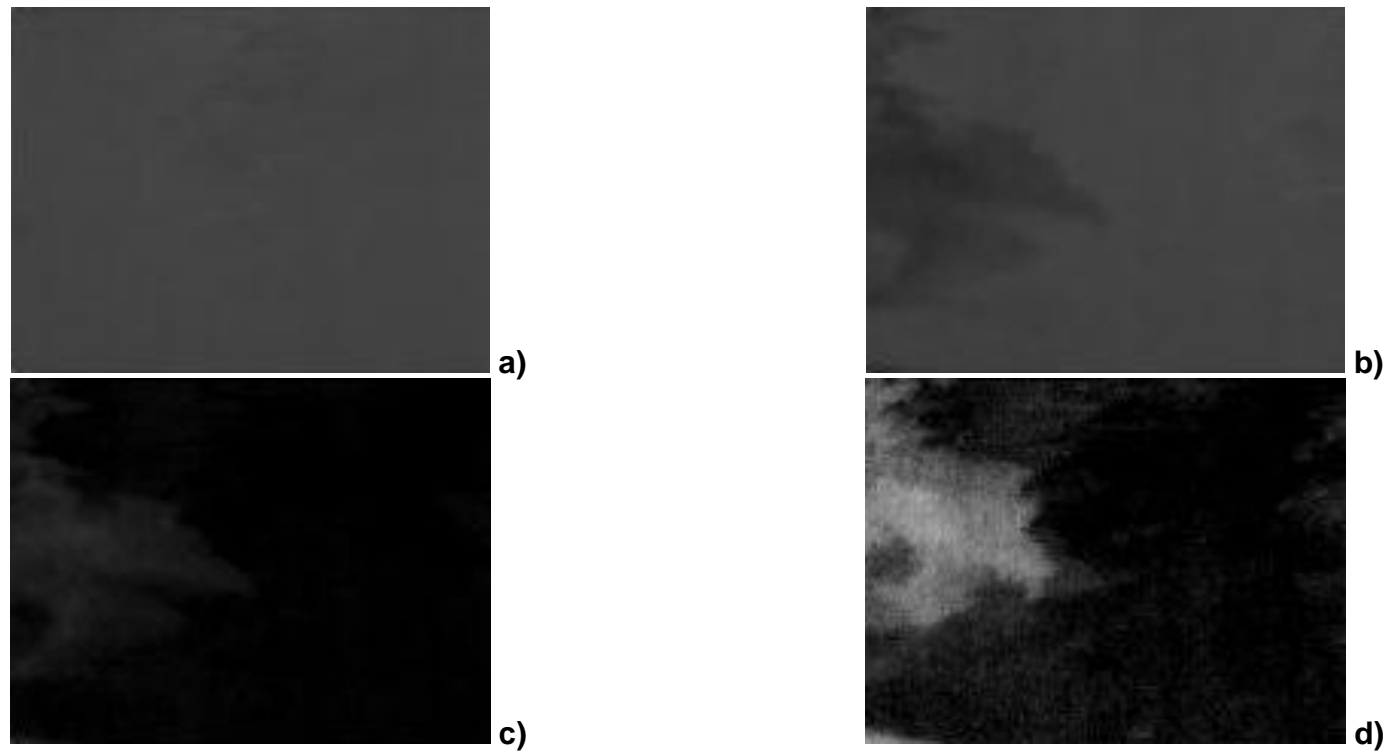

Fig. 3. Infrared and resulting images during the laboratory analysis with wind. a)averaged reference thermogram; b)thermogram acquisition after hydrocarbon injection; c) result of the single image analysis; d)result of integrated $k$ images analysis. 
Fig. 4 shows the analysis result in the case of real conditions. The acquisition has been performed during a sunny day, with an external temperature of $16^{\circ} \mathrm{C}$, with a wind speed equal to $4 \mathrm{~ms}^{-1}$.
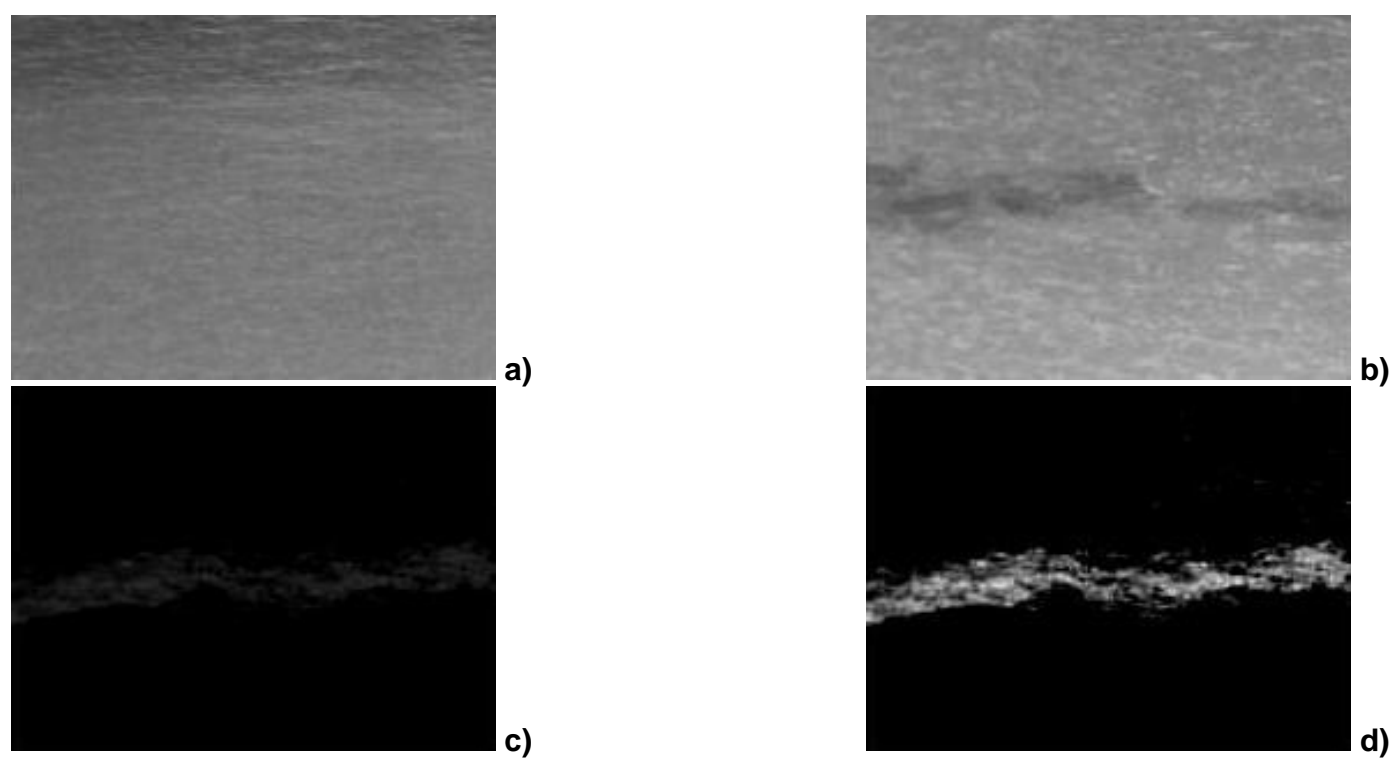

Fig. 3. Infrared and resulting images during the on-field analysis. a)averaged reference thermogram; b)thermogram acquisition after hydrocarbon injection; c)result of the single image analysis,

d)result of integrated $k$ images analysis.

\section{Discussion and conclusions}

A new automated analysis algorithm has been designed, in order to identify the hydrocarbons presence on the sea surface. The algorithm basic theoretical idea is that as an hydrocarbon film is present on the water surface, it tends to stay compact over time, avoiding dispersions. Therefore, the implementation of image integration permits to reduce the random external noise, increasing the hydrocarbons typical gradient.

The integration interval evaluation required several tests, in which different external conditions have been simulated. Results have shown the preliminary effectiveness of the proposed algorithm, in laboratory conditions. As for real sea surface analysis, good results have been obtained in good weather conditions (low wind, both with sun or rain), in respect to traditional techniques.

The main limitation of the proposed test is the difficult application in cases of strong wind or rough sea, for which more tests are needed to improve the algorithm capability of detecting pollutant.

\section{REFERENCES}

[1] Eddy Melton R., Sabol B.M., "A Therman IR Processing Technique for Detecting and Tracking Objects in Motion", ITC121A Inframation conference, 2007.

[2] Distefano G., "Use of IR and visible station to monitor and prevent sea coast and sea water pollution in high risk sites". Inframation 2009 conference, LasVegas, 2009.

[3] Wei-Chuan S., Ballard Andrews A., "Infrared contrast of crude-oil-covered water surfaces," Opt. Lett.33, 30193021, 2008.

[4] Goodman R.H., "The remote sensing of oil slicks", Lodge AE, ed., (John Wiley and Sons, Chichester, UK, 1989), pp. 39-65. 